\title{
Ternary eutectic growth of Ag-Cu-Sb alloy within ultrasonic field
}

\author{
ZHAI Wei, HONG ZhenYu \& WEI BingBo ${ }^{\dagger}$ \\ Department of Applied Physics, Northwestern Polytechnical University, Xi'an 710072, China
}

The liquid to solid transformation of ternary $\mathrm{Ag}_{42.4} \mathrm{Cu}_{21.6} \mathrm{Sb}_{36}$ eutectic alloy was accomplished in an ultrasonic field with a frequency of $35 \mathrm{kHz}$, and the growth mechanism of this ternary eutectic was examined. Theoretical calculations predict that the sound intensity in the liquid phase at the solidification interface increases gradually as the interface moves up from the sample bottom to its top. The growth mode of $(\varepsilon+\theta+\mathrm{Sb})$ ternary eutectic exhibits a transition of "divorced eutecticmixture of anomalous and regular structures-regular eutectic" along the sample axis due to the inhomogeneity of sound field distribution. In the top zone with the highest sound intensity, the cavitation effect promotes the three eutectic phases to nucleate independently, while the acoustic streaming efficiently suppresses the coupled growth of eutectic phases. In the meantime, the ultrasonic field accelerates the solute transportation at the solid-liquid interface, which reduces the solute solubility of eutectic phases.

ternary eutectic, eutectic growth, ultrasonic field, cavitation effect, acoustic streaming

Solidification within ultrasonic field has been extensively studied in the field of material physics $^{[1-5]}$. The effects of ultrasound on solidification include the following aspects. (i) Nucleation promotion. Ohsaka et al. ${ }^{[1]}$ reported that ultrasound prematurely terminates the undercooling of levitated succinonitrile drops by initiating nucleation. Hunt et al. ${ }^{[6]}$ observed that the collapse of the cavitation bubbles is the major factor to cause heterogeneous nucleation in Benzene-water solution. (ii) Grain refining and coarsening. Ultrasound leads to grain refinement through suppressing columnar dendrite and promoting the formation of equaixed grain ${ }^{[7]}$. Although the grain size is decreased by ultrasound, the secondary dendritic arm spacing is sometimes increased remarkably ${ }^{[8,9]}$. (iii) Segregation suppression. Ultrasonic field may eliminate or suppress the macro- and microsegregation during solidification, producing microstructures with uniform solute distribution ${ }^{[7,10]}$.

Most research work has been mainly focused on the effects of ultrasound field on the microstructure evolution. The question is whether it is reasonable to determine the structural character-

Received February 11, 2007; accepted May 23, 2007 doi: $10.1007 / \mathrm{s} 11433-007-0043-9$

${ }^{\dagger}$ Corresponding author (email: bbwei@nwpu.edu.cn)

Supported by the National Natural Science Foundation of China (Grant Nos. 50121101 and 50395105) 
istics of the whole sample according to its local microstructure changes. In fact, when a wave travels through a solidifying alloy melt, reflection and transmission always take place because of the existence of liquid/solid interface, which will probably arouse various effects at different zones of the alloy sample. On the other hand, investigations are mainly focused on the single dendritic or binary eutectic growth. The solidification mechanisms are much more complicated when multiphase transformation occurs, and there have been few reports in the literature up to now.

The ternary Ag-Cu-Sb eutectic alloy, whose solidification process involves the nucleation and growth of two intermetallic compounds and one faceted solid solution phase, is a typical ternary eutectic system of low melting point. The objective of the present work is to examine the microstructure transition of $\mathrm{Ag}_{42.4} \mathrm{Cu}_{21.6} \mathrm{Sb}_{36}$ eutectic alloy along the direction of wave propagation and investigate the growth mechanism of ternary eutectic within the ultrasonic field.

\section{Experimental procedure}

The experiments were performed in a solidification apparatus incorporated with ultrasonic generator. $\mathrm{Ag}_{42.4} \mathrm{Cu}_{21.6} \mathrm{Sb}_{36}$ alloy samples were prepared from $99.9 \% \mathrm{Ag}, 99.99 \% \mathrm{Cu}$ and $99.99 \% \mathrm{Sb}$, and each sample was $\Phi 10 \times 15 \mathrm{~mm}$ in size, contained in a $\Phi 10 \times 14 \times 40 \mathrm{~mm}$ quartz crucible. The sample was heated by an electrical resistance furnace in argon atmosphere, and its temperature was monitored by a NiCr-NiSi thermocouple. The ultrasonic generator consists of two parts: a $\mathrm{KNbO}_{3}$ piezoelectric transducer with a resonant frequency of $35 \mathrm{kHz}$ and an emitter with an end plane of $\Phi 9.9 \times 4.0 \mathrm{~mm}$. When temperature dropped to the eutectic temperature of $699 \mathrm{~K}$, the ultrasonic transducer was turned on and the longitudinal ultrasonic wave was introduced into the alloy melt until it solidified completely.

After experiments, the solidified samples were vertically sectioned, mounted in epoxy resin, polished, and etched with a solution of $5 \mathrm{~g} \quad \mathrm{FeCl}_{3}+2 \mathrm{~mL} \mathrm{HCl}+1 \mathrm{~mL} \mathrm{HNO}_{3}+97 \mathrm{~mL} \mathrm{H}_{2} \mathrm{O}$. The microstructures of solidified samples were analyzed by a Rigaku D/max $2500 \mathrm{X}$-ray diffractometer, a Zeiss Axiovert 200 MAT optical microscope and an FEI Sirion 200 scanning electron microscope.

\section{Results and discussion}

\subsection{Acoustic field distribution within solidifying alloy}

During the liquid-solid transformation of $\mathrm{Ag}_{42.4} \mathrm{Cu}_{21.6} \mathrm{Sb}_{36}$ ternary alloy, nucleation is initiated at the sample bottom. After that, the solid/liquid interface gradually moves upwards until the whole sample solidifies. In order to get a better understanding of the growth mechanism of $\mathrm{Ag}-\mathrm{Cu}-\mathrm{Sb}$ ternary eutectic within the ultrasonic field, the acoustic field distribution within the solidifying alloy melt is calculated. To simplify the problem, several assumptions are made as follows: (i) the solid and liquid phases are separated by a planar interface, and (ii) the wave propagation in the alloy melt shows a plane wave mode. Figure 1 presents schematically the sound wave propagation in a solidifying sample. The coordinate system is established in such a way that the position of emitter/liquid interface is chosen as the origin $O . h$ denotes the length along the direction of wave propagation, and $d$ is the location of liquid/solid interface, which moves upwards during solidification. $h=1.5 \times 10^{-2} \mathrm{~m}$ and $1.6 \times 10^{-2} \mathrm{~m}$ are the positions of solid/crucible and crucible/air interfaces, respectively. The acoustic field is analyzed by considering acoustic reflection and transmission at all kinds of interfaces existing in the propagating direction of the incident plane wave. 


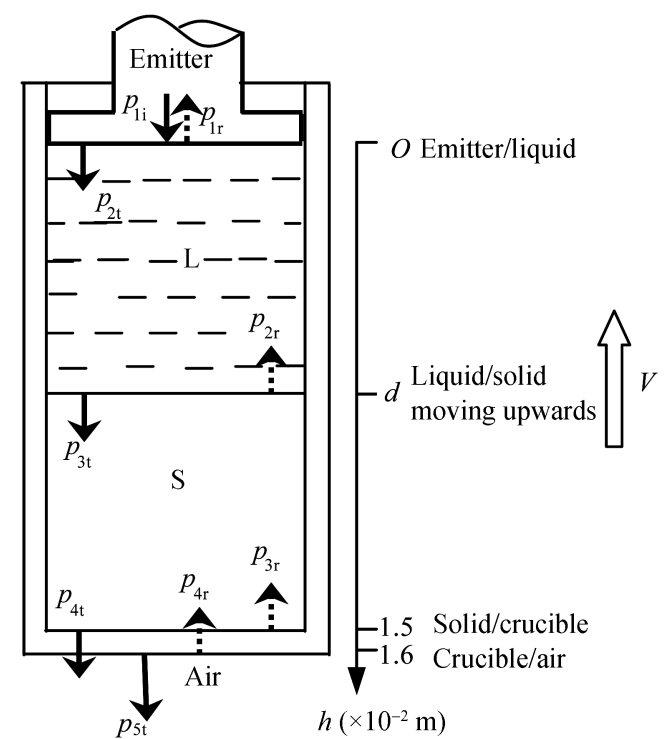

Figure 1 Illustration of sound wave propagation within the solidifying sample.

When an incident plane wave $\left(p_{1 \mathrm{i}}, v_{1 \mathrm{i}}\right)$ travels vertically through the emitter/melt interface, one part of this wave returns to the emitter, and the other part transmits into the alloy melt. The reflected and transmitted waves can be written as $\left(p_{1 \mathrm{r}}, v_{1 \mathrm{r}}\right)$ and $\left(p_{2 \mathrm{t}}, v_{2 \mathrm{t}}\right)$, respectively. Similarly, the transmission and reflection occur at the following interfaces: the liquid/solid interface $(h=d)$ with the reflected wave $\left(p_{2 \mathrm{r}}, v_{2 \mathrm{r}}\right)$ and the transmitted wave $\left(p_{3 \mathrm{t}}, v_{3 \mathrm{t}}\right)$, then the interface of solid alloy/crucible $\left(h=1.5 \times 10^{-2} \mathrm{~m}\right)$ with the reflected wave $\left(p_{3 \mathrm{r}}, v_{3 \mathrm{r}}\right)$ and the transmitted wave $\left(p_{4 \mathrm{t}}, v_{4 \mathrm{t}}\right)$, and the crucible/air interface $\left(h=1.6 \times 10^{-2} \mathrm{~m}\right)$ with the reflected wave $\left(p_{4 \mathrm{r}}, v_{4 \mathrm{r}}\right)$ and the transmitted wave $\left(p_{5 \mathrm{t}}\right.$, $\left.v_{5 t}\right)$. Assuming that the air is an infinite medium in which no reflection takes place, each wave can be expressed as follows:

$$
\begin{gathered}
\left\{\begin{array}{l}
p_{1 \mathrm{i}}=p_{1 \mathrm{ia}} \mathrm{e}^{\mathrm{j}\left(w t-k_{1} h\right),} \\
v_{1 \mathrm{i}}=v_{1 \mathrm{ia}} \mathrm{e}^{\mathrm{j}\left(w t-k_{1} h\right),}
\end{array}\right. \\
\left\{\begin{array}{l}
p_{1 \mathrm{r}}=p_{1 \mathrm{ra}} \mathrm{e}^{\mathrm{j}\left(w t+k_{1} h\right),} \\
v_{1 \mathrm{r}}=v_{1 \mathrm{ra}} \mathrm{e}^{\mathrm{j}\left(w t+k_{1} h\right),}
\end{array}\right. \\
\left\{\begin{array}{l}
p_{2 \mathrm{t}}=p_{2 \mathrm{ta}} \mathrm{e}^{\mathrm{j}\left(w t-k_{2} h\right),} \\
v_{2 \mathrm{t}}=v_{2 \mathrm{ta}} \mathrm{e}^{\mathrm{j}\left(w t-k_{2} h\right),}
\end{array}\right. \\
\left\{\begin{array}{l}
p_{2 \mathrm{r}}=p_{2 \mathrm{ta}} \mathrm{e}^{\mathrm{j}\left(w t+k_{2} h\right),} \\
v_{2 \mathrm{r}}=v_{2 \mathrm{ta}} \mathrm{e}^{\mathrm{j}\left(w t+k_{2} h\right),} \\
p_{3 \mathrm{t}}=p_{3 \mathrm{ta}} \mathrm{e}^{\mathrm{j}\left(w t-k_{3}(h-d)\right),} \\
v_{3 \mathrm{t}}=v_{3 \mathrm{ta}} \mathrm{e}^{\mathrm{j}\left(w t-k_{3}(h-d)\right),}
\end{array}\right. \\
\left\{\begin{array}{l}
p_{3 \mathrm{r}}=p_{3 \mathrm{ra}} \mathrm{e}^{\mathrm{j}\left(w t+k_{3}(h-d)\right),} \\
v_{3 \mathrm{r}}=v_{3 \mathrm{ra}} \mathrm{e}^{\mathrm{j}\left(w t+k_{3}(h-d)\right),}
\end{array}\right.
\end{gathered}
$$




$$
\begin{aligned}
& \left\{\begin{array}{l}
p_{4 \mathrm{t}}=p_{4 \mathrm{ta}} \mathrm{e}^{\mathrm{j}\left(w t-k_{4}(h-1.5)\right),} \\
v_{4 \mathrm{t}}=v_{4 \mathrm{ta}} \mathrm{e}^{\mathrm{j}\left(w t-k_{4}(h-1.5)\right),}
\end{array}\right. \\
& \left\{\begin{array}{l}
p_{4 \mathrm{r}}=p_{4 \mathrm{ra}} \mathrm{e}^{\mathrm{j}\left(w t+k_{4}(h-1.5)\right)} \\
v_{4 \mathrm{r}}=v_{4 \mathrm{ra}} \mathrm{e}^{\mathrm{j}\left(w t+k_{4}(h-1.5)\right)},
\end{array}\right. \\
& \left\{\begin{array}{l}
p_{5 \mathrm{t}}=p_{5 \mathrm{ta}} \mathrm{e}^{\mathrm{j}\left(w t-k_{5}(h-1.6)\right),} \\
v_{5 \mathrm{t}}=v_{5 \mathrm{ta}} \mathrm{e}^{\mathrm{j}\left(w t-k_{5}(h-1.6)\right)},
\end{array}\right.
\end{aligned}
$$

where $p_{\text {lia }}, p_{\text {lra }}, p_{2 \text { ta }}, p_{2 \text { ra }}, p_{3 \text { ta }}, p_{3 \text { ra }}, p_{4 \text { ta }}, p_{4 \text { ra }}$ and $p_{5 \text { ta }}$ are the sound pressure amplitudes of waves; $v_{\text {lia, }}$ $v_{\text {lra }}, v_{2 \text { ta }}, v_{2 \text { ra }}, v_{3 \text { ta }}, v_{3 \text { ra }}, v_{4 \text { ta }}, v_{4 \text { ra }}$ and $v_{5 \text { ta }}$ are the normal particle velocity amplitudes; $t$ is the time; $\omega=$ $219800 \mathrm{rad} / \mathrm{s}$ is the angular frequency; $k_{n}(n=1,2,3,4,5)$ is the wavenumber of each medium. According to the acoustic boundary conditions with respect to the continuity of sound pressure and continuity of normal velocity at interfaces, eqs. (10)-(13) can be obtained.

At $h=0$,

$$
\left\{\begin{array}{l}
p_{1 \mathrm{ia}}+p_{1 \mathrm{ra}}=p_{2 \mathrm{ta}}+p_{2 \mathrm{ra}} \\
v_{\text {lia }}+v_{1 \mathrm{ra}}=v_{2 \mathrm{ta}}+v_{2 \mathrm{ra}}
\end{array}\right.
$$

at $h=d$,

$$
\left\{\begin{array}{l}
p_{2 \mathrm{ta}} \mathrm{e}^{-\mathrm{j} k_{2} d}+p_{2 \mathrm{ra}} \mathrm{e}^{\mathrm{j} k_{2} d}=p_{3 \mathrm{ta}}+p_{3 \mathrm{ra}} \\
v_{2 \mathrm{ta}} \mathrm{e}^{-\mathrm{j} k_{2} d}+v_{2 \mathrm{ra}} \mathrm{e}^{\mathrm{j} k_{2} d}=v_{3 \mathrm{ta}}+v_{3 \mathrm{ra}}
\end{array}\right.
$$

at $h=1.5 \times 10^{-2} \mathrm{~m}$,

at $h=1.6 \times 10^{-2} \mathrm{~m}$,

$$
\left\{\begin{array}{l}
p_{3 \mathrm{ta}} \mathrm{e}^{-\mathrm{j} k_{3}(1.5-d)}+p_{3 \mathrm{ra}} \mathrm{e}^{\mathrm{j} k_{3}(1.5-d)}=p_{4 \mathrm{ta}}+p_{4 \mathrm{ra}} \\
v_{3 \mathrm{ta}} \mathrm{e}^{-\mathrm{j} k_{3}(1.5-d)}+v_{3 \mathrm{ra}} \mathrm{e}^{\mathrm{j} k_{3}(1.5-d)}=v_{4 \mathrm{ta}}+v_{4 \mathrm{ra}}
\end{array}\right.
$$

$$
\left\{\begin{array}{l}
p_{4 \mathrm{ta}} \mathrm{e}^{-\mathrm{j} k_{4}(1.6-1.5)}+p_{4 \mathrm{ra}} \mathrm{e}^{\mathrm{j} k_{4}(1.6-1.5)}=p_{5 \mathrm{ta}}, \\
v_{4 \mathrm{ta}} \mathrm{e}^{-\mathrm{j} k_{4}(1.6-1.5)}+v_{4 \mathrm{ra}} \mathrm{e}^{\mathrm{j} k_{4}(1.6-1.5)}=v_{5 \mathrm{ta}}
\end{array}\right.
$$

Since all of the waves show a plane wave mode, the relationship between sound pressure and normal velocity is expressed in eqs. (14) and (15):

$$
\begin{gathered}
v_{n \text { ia }}=p_{n \text { ia }} / R_{n}, \\
v_{n \text { ra }}=-p_{n \text { ra }} / R_{n},
\end{gathered}
$$

where $R_{n}=\rho_{n} c_{n}(n=1,2,3,4,5)$ is the acoustic impedance of each medium; $\rho_{n}$ and $c_{n}$ are the density and sound speed, respectively.

The incident sound pressure amplitude

$$
p_{\text {lia }}=8.0 \times 10^{5} \mathrm{~Pa} .
$$

Substituting eqs. (10)-(16) into eqs. (1) - (9), the sound pressure amplitude of each wave can be achieved. Then, the sound pressure within the solidifying alloy melt is expressed as

$$
p_{\text {alloy }}=\left\{\begin{array}{l}
p_{2 \mathrm{t}}+p_{2 \mathrm{r}}=p_{2 \mathrm{ta}} \mathrm{e}^{\mathrm{j}\left(w t-k_{2} h\right)}+p_{2 \mathrm{ra}} \mathrm{e}^{\mathrm{j}\left(w t+k_{2} h\right)}, \quad h \leqslant d, \\
p_{3 \mathrm{t}}+p_{3 \mathrm{r}}=p_{3 \mathrm{ta}} \mathrm{e}^{\mathrm{j}\left(w t-k_{3}(h-d)\right)}+p_{3 \mathrm{ra}} \mathrm{e}^{\mathrm{j}\left(w t+k_{3}(h-d)\right)}, \quad d<h \leqslant 1.5 \times 10^{-2} \mathrm{~m} .
\end{array}\right.
$$

According to eq. (17), the sound pressure amplitude distribution along $h$ direction with the 
variation of liquid/solid interface location $d$ is calculated, and the result is shown in Figure 2. The physical parameters used in calculations are listed in Table 1.

Table 1 Physical parameters used in calculations

\begin{tabular}{ccccc}
\hline Medium & Density $\rho\left(\mathrm{kg} / \mathrm{m}^{3}\right)$ & Sound velocity $v(\mathrm{~m} / \mathrm{s})$ & Acoustic impedance $R\left(\times 10^{7} \mathrm{~N} \cdot \mathrm{s} / \mathrm{m}^{3}\right)$ & Wave number $k(\mathrm{rad} / \mathrm{m})$ \\
\hline Emitter & 7800 & 6100 & 4.77 & 36 \\
Liquid alloy & 8018 & 2620 & 2.10 & 83 \\
Solid alloy & 8771 & 3480 & 3.05 & 63 \\
Quartz crucible & 2700 & 5570 & 1.50 & 39 \\
Air & 1.21 & 344 & $4.16 \times 10^{-5}$ & 148 \\
\hline
\end{tabular}

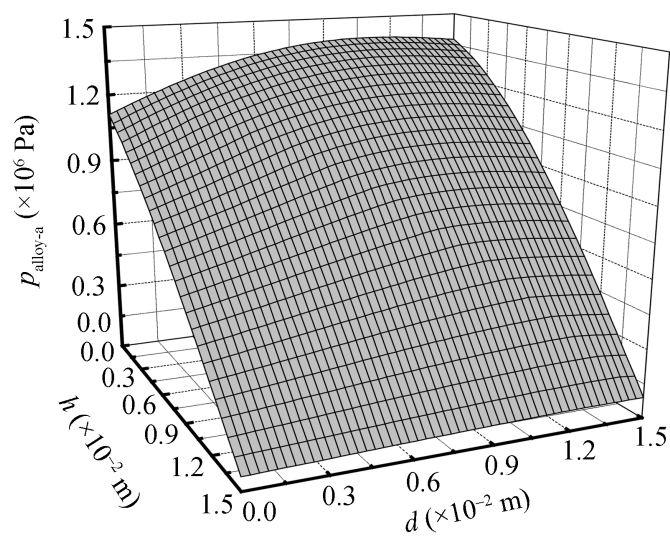

Figure 2 Sound field distribution within $\mathrm{Ag}_{42.4} \mathrm{Cu}_{21.6} \mathrm{Sb}_{36}$ alloy sample.

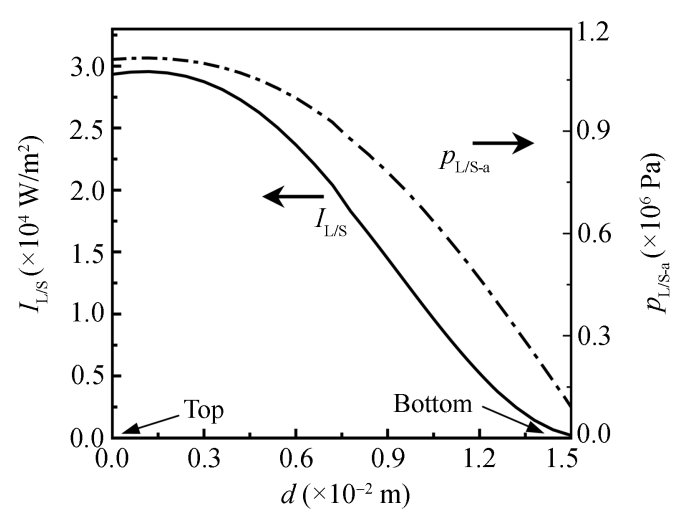

Figure 3 Sound intensity and sound pressure in the liquid phase at the solidification interface.

Clearly, the further the location is away from the sound source, the smaller the value of the pressure amplitude is. Even at the same position of the alloy sample, the pressure amplitude varies with the location change of the solid/liquid interface. Since the sound intensity may be a dominant factor influencing microstructural morphology, the sound intensity and sound pressure in the liquid phase at the solid/liquid interface are also calculated, as plotted in Figure 3. It can be seen that the sound intensity $I_{\mathrm{L} / \mathrm{S}}$ and the sound pressure $p_{\mathrm{L} / \mathrm{S} \text {-a }}$ become higher as the liquid/solid interface moves upwards. At the sample bottom, the sound intensity is nearly zero, suggesting that the solidification process may not be affected by the ultrasonic field here. At the sample top, the sound intensity reaches $2.9 \times 10^{4} \mathrm{~W} / \mathrm{m}^{2}$. These results indicate that the effect of the ultrasonic field on the solidification process maybe weakens from the top to the bottom of $\mathrm{Ag}_{42.4} \mathrm{Cu}_{21.6} \mathrm{Sb}_{36}$ alloy sample.

The above model reflects how a plane wave propagates in the alloy melt. However, due to the fact that the emitter diameter is slightly smaller than that of the crucible diameter, a little part of sound wave energy may deviate from $h$ direction. Thus, the actual sound pressure and sound intensity may be smaller than the present calculated results.

\subsection{Microstructural characteristics of $\mathrm{Ag}_{42.4} \mathrm{Cu}_{21.6} \mathrm{Sb}_{36}$ eutectic alloy}

The fact that ultrasound can influence the phase transformation and the formation of intermetallic compounds has been confirmed by many researchers ${ }^{[11]}$. Hence, X-ray diffraction (XRD) analyses are necessary to check whether there are new metastable phases formed within the ultrasonic field. As illustrated in Figure 4, both of the samples, solidified under static and ultrasonic conditions, are 
composed of $\theta\left(\mathrm{Cu}_{2} \mathrm{Sb}\right), \varepsilon\left(\mathrm{Ag}_{3} \mathrm{Sb}\right)$, and ( $\left.\mathrm{Sb}\right)$ phases. The ultrasonic field here does not change the phase constitution of $\mathrm{Ag}_{42.4} \mathrm{Cu}_{21.6}$ $\mathrm{Sb}_{36}$ ternary eutectic alloy.

Figure 5 presents the growth morphologies of $\mathrm{Ag}_{42.4} \mathrm{Cu}_{21.6} \mathrm{Sb}_{36}$ ternary eutectic alloy along the sample axis. Three distinct zones appear from the sample top to its bottom, named zone I, II, and III, which take up $40 \%, 44 \%$ and $16 \%$ of the total sample height, respectively. The divorced $(\theta+\varepsilon+\mathrm{Sb})$ ternary eutectic shown in Figure 5(a) is the unique microstructure of zone I. Both $(\mathrm{Sb})$ and $\varepsilon$ phases exhibit an equiaxed shape, and $\theta$ phase shows a short strip shape. The three eutectic phases distribute homoge-

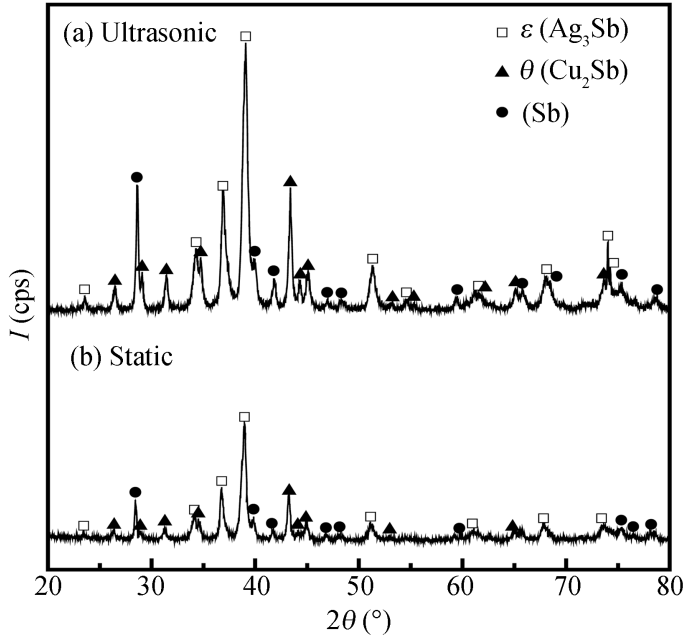

Figure 4 XRD patterns of $\mathrm{Ag}_{42.4} \mathrm{Cu}_{21.6} \mathrm{Sb}_{36}$ eutectic alloy. neously, and grow in a divorced eutectic mode. In zone II, the microstructure is characterized by a mixture of anomalous and regular $(\theta+\varepsilon+\mathrm{Sb})$ ternary eutectics. In anomalous eutectic, $(\mathrm{Sb})$ phase exhibits a branched growth. In addition, there is a little fraction of regular $(\theta+\varepsilon+\mathrm{Sb})$ ternary eutectic surrounding $\theta$ phase, as shown in Figure 5(b). In zone III, the microstructure consists of a small amount of $(\theta+\varepsilon)$ pseudobinary eutectic and a large amount of regular $(\theta+\varepsilon+\mathrm{Sb})$ ternary eutectic. The regular $(\theta+\varepsilon+\mathrm{Sb})$ ternary eutectic grows surrounding primary $\theta$ phase that has an obvious preferred crystallographic direction. In the regular $(\theta+\varepsilon+\mathrm{Sb})$ ternary eutectic, $\theta$ and $\varepsilon$ phases grow in a parallel way, and the faceted ( $\mathrm{Sb}$ ) phase shows an angle with them. This kind of microstructure is almost the same as the solidification structure under a static condition ${ }^{[12]}$, implying that the solidification process here is not influenced by the ultrasonic field actually. This is in good agreement with the calculated results that the sound intensity at the sample bottom is nearly

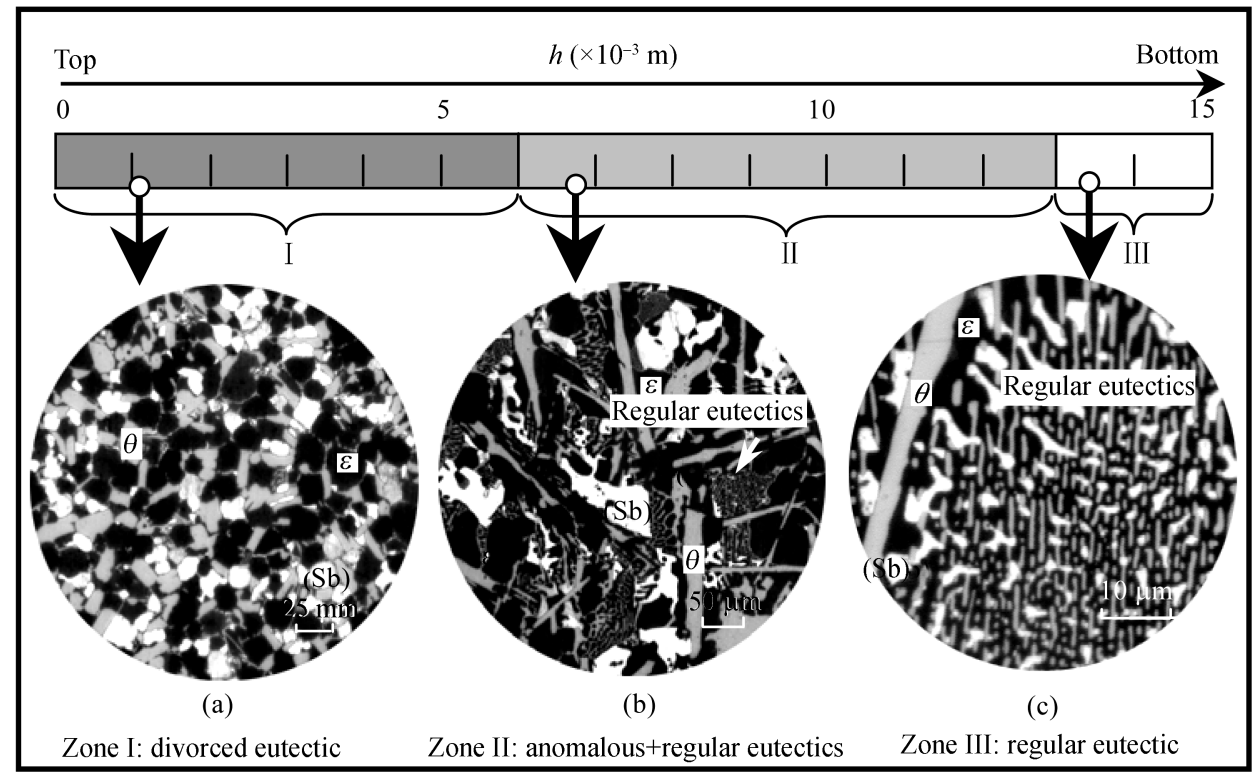

Figure 5 Growth morphologies of $\mathrm{Ag}_{42.4} \mathrm{Cu}_{21.6} \mathrm{Sb}_{36}$ ternary eutectic along the sample axis. 
zero. It should be pointed out that the growth morphology changes gradually along the sample axis, and there are no obvious boundaries among the three different zones.

The length-width ratios of $\theta$ phase in the three different zones are measured, as illustrated in Figure 6. Evidently, this slenderness ratio reduces sharply from 24 to 2 as the sound intensity increases, which indicates that the preferred growth of $\theta$ phase is greatly restrained within the ultrasonic field. Figure 7 presents the volume fraction of anomalous $(\theta+\varepsilon+\mathrm{Sb})$ ternary eutectic versus sound intensity. It reveals that the sound intensity has a drastic effect on the volume fraction of anomalous eutectic. With the increase of sound intensity, the volume fraction of anomalous eutectic increases conspicuously. Through the growth morphology transition, it can be proved that the effect of the acoustic field on these three zones weakens because sound intensity decreases from the top to the bottom of the alloy sample.

EDS method is applied to determining the solute contents in three eutectic phases of different zones, and the results are shown in Figure 8. Apparently, the contents of solute $\mathrm{Cu}$ and $\mathrm{Ag}$ in $(\mathrm{Sb})$ phase, solute $\mathrm{Cu}$ in $\varepsilon\left(\mathrm{Ag}_{3} \mathrm{Sb}\right)$ phase, and solute $\mathrm{Ag}$ in $\theta\left(\mathrm{Cu}_{2} \mathrm{Sb}\right)$ phase decrease with the increase of sound intensity, especially for $\varepsilon\left(\mathrm{Ag}_{3} \mathrm{Sb}\right)$ and $(\mathrm{Sb})$ phases. Both of them become pure in zone I. This implies that the ultrasonic field influences the solute distribution process during the growth of eutectic phases by promoting the solute diffusion in liquid phase, which reduces the thickness of the boundary layer and depresses the solute enrichment at the liquid/solid interface. In other words, the ultrasonic field lowers the solute solubility of each eutectic phase.

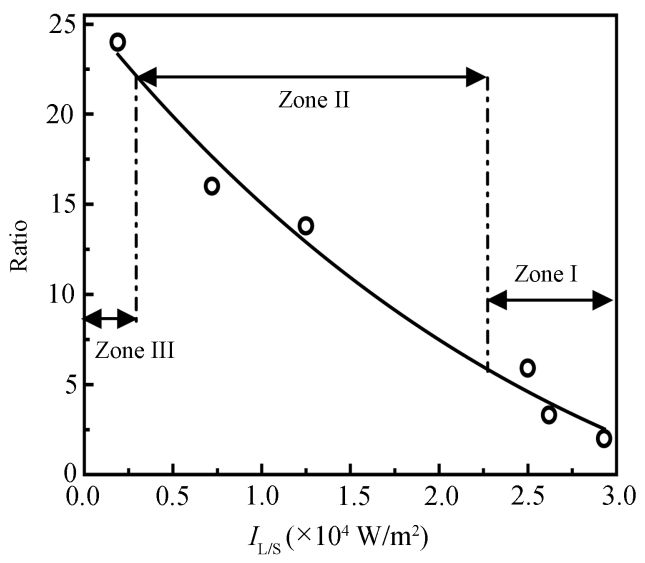

Figure 6 Slenderness ratio of $\theta$ phase under different sound intensities.

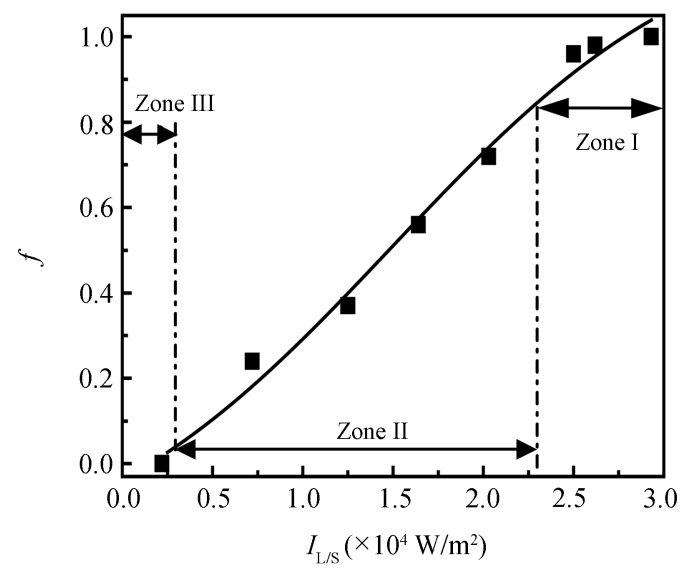

Figure 7 Volume fraction of $(\theta+\varepsilon+\mathrm{Sb})$ ternary anomalous eutectic versus sound intensity.

\subsection{Ternary eutectic growth within ultrasonic field}

According to the above analyses, it can be summarized that the effect of the ultrasonic field on $\mathrm{Ag}_{42.4} \mathrm{Cu}_{21.6} \mathrm{Sb}_{36}$ ternary eutectic growth mechanism depends on sound intensity to a great extent.

When a strong ultrasound wave travels through the alloy melt, it can generate cavities and small bubbles. These tiny bubbles are inherently unstable and can continuously grow by absorbing the gas from the surrounding melt. If the sound pressure reaches the threshold value, the bubbles can collapse drastically and generate a local high pressure. Theoretical calculations suggest that the threshold value of sound pressure increases with the decrease of the bubble radius. Given a very small bubble of $R=5 \mu \mathrm{m}$ in $\mathrm{Ag}_{42.4} \mathrm{Cu}_{21.6} \mathrm{Sb}_{36}$ alloy melt, the threshold sound pressure $P_{\mathrm{B}}$ is $1.1 \times 10^{6}$ $\mathrm{Pa}$. According to Figure 3 , the sound pressure amplitudes in zone I are higher than this value, which 

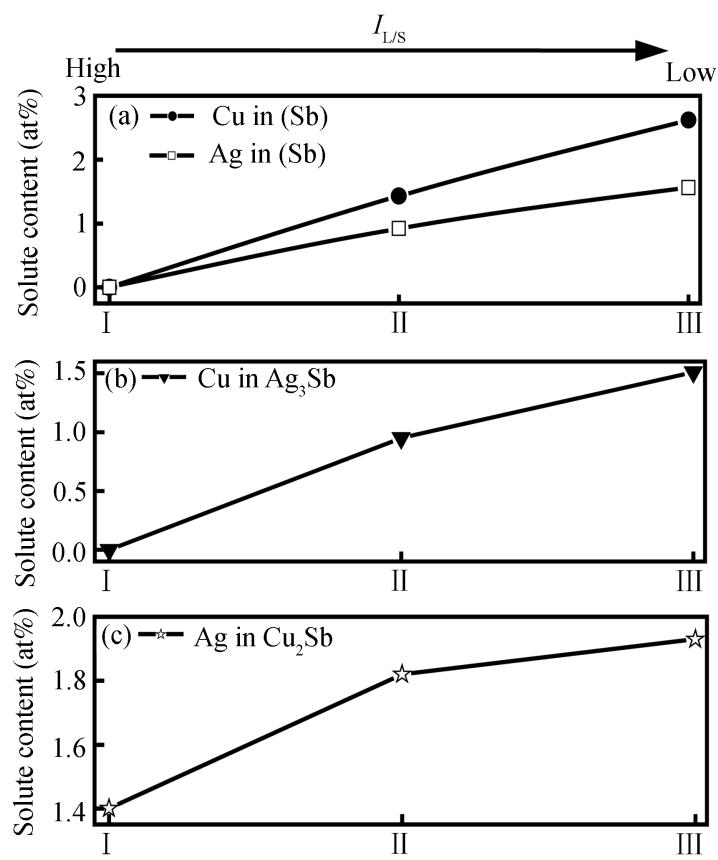

Figure 8 Solute contents of three eutectic phases in different zones.

means that cavitation tends to take place in this zone during solidification. On the other hand, due to the existence of sound pressure gradient, acoustic streaming forms and can affect the solidification process by stirring the alloy melt.

In zone I, the formation of divorced eutectic is mainly caused by cavitation effect and acoustic streaming. It is well known that the cavitation effect produces ultrahigh pressure (which can reach $5 \mathrm{GPa}^{[13,14]}$ ) after the collapse of microbbules and initiates nucleation in undercooled liquid by raising the local melting point and lowering the local activation energy for nucleation. The rise of melting point $T_{\mathrm{m}}$ with pressure $P$ can be expressed by Clausius-Clapeyron equation:

$$
T_{\mathrm{m}}=T_{\mathrm{n}}+\frac{T_{\mathrm{n}} \Delta V}{\Delta H_{\mathrm{m}}}\left(P-P_{0}\right),
$$

where $T_{\mathrm{n}}$ is the melting point at atmospheric pressure, $P_{0} ; \Delta V$ and $\Delta H_{\mathrm{m}}$ are the volume change and enthalpy change during liquid-solid transformation, respectively. Assuming that the high pressure is up to $5 \mathrm{GPa}$, the local melting temperature of $\mathrm{Ag}_{42.4} \mathrm{Cu}_{21.6} \mathrm{Sb}_{36}$ alloy is elevated by $75 \mathrm{~K}$, which makes the undercooling level and nucleation probability in the vicinity of cavitation site much higher than those in bulk alloy melt ${ }^{[15]}$. Thus, the three eutectic phases can nucleate dependently. In the meantime, the maximum possible velocity of acoustic streaming is given as $v=\sqrt{2} \pi f a$ ( $a$ denotes the amplitude of vibration), and this means that acoustic streaming with a velocity of $v=$ $4.66 \mathrm{~m} / \mathrm{s}$ can be produced in the alloy melt. This enhances the solute interdiffusion among eutectic phases, and induces disturbances and instabilities at the solid-liquid interface. Therefore, the coupled growth mechanism under the static condition is interrupted to a certain degree and the anomalous eutectic growth mode takes place.

With the decrease of sound intensity, the acoustic streaming and the acoustic cavitation effect are reduced rapidly. Thus, in some micro-areas, the three eutectic phases grow in a cooperative way and the growth morphology exhibits a mixture of regular and anomalous $(\varepsilon+\theta+\mathrm{Sb})$ ternary eutec- 
tics. At the sample bottom, the sound intensity is too low to influence the solidification process, and the growth mode is a regular eutectic growth.

\section{Conclusions}

(1) There exists a sound pressure gradient within the solidifying $\mathrm{Ag}_{42.4} \mathrm{Cu}_{21.6} \mathrm{Sb}_{36}$ alloy melt. Both of the sound pressure amplitude and the sound intensity in liquid phase adjacent to the solidification front increase as the liquid/solid interface moves upwards.

(2) $(\varepsilon+\theta+\mathrm{Sb})$ ternary eutectic experiences a growth morphology transition of "divorced eutectic - mixture of anomalous and regular eutectics — regular eutectic" along the sample axis due to the inhomogeneity of the ultrasonic field.

(3) The solute content in each eutectic phase decreases with the increase of sound intensity, owing to the enhanced solute diffusion in liquid phase resulting from acoustic streaming.

(4) The formation of divorced $(\varepsilon+\theta+\mathrm{Sb})$ ternary eutectic is mainly caused by cavitation and acoustic streaming effects. The cavitation effect promotes eutectic phases to nucleate independently, while the acoustic streaming efficiently suppresses the coupled growth of eutectic phases.

The authors are grateful to Ms. Wang Weili, Ms. Ruan Ying, Mr. Wang Haipeng and Dr. Yao Wenjing for their help with experimental work and discussions.

1 Ohsaka K, Trinh E H. Dynamic nucleation of ice induced by a single stable cavitation bubble. Appl Phys Lett, 1998, 73: $129-132$

2 Zhai W, Hong Z Y, Xie W J, et al. Solidification characteristics of Pb-Sb hypereutectic alloy within ultrasonic field. Chin Sci Bull, 2007, 52(6): 844-848

3 Chamuel J R. Transient Schlte wave transmission along liquid-solid interfaces. J Acoust Soc Am, 1988, 83(4): 1336-1353

4 Zhang X, Inada T, Tezuka A. Ultrasonics induced nucleation of ice in water containing air bubbles. Ultrason Sonochem, 2003, 10(2): 71-76

5 Lee C P, Wang T G. The effects of pressure on the nucleation rate of undercooled liquid. J Appl Phys, 1992, 71: 5721-5723

6 Hunt J D, Jackson K A. Nucleation of solid in an undercooled liquid by cavitation. J Appl Phys, 1966, 37: 254-257

7 Wei B. Unidirectional dendritic solidification under longitudinal resonant vibration. Acta Metall Mater, 1992, 40(1): $2739-2751$

8 Chow R, Blindt R, Chivers R, et al. The sonocrystallisation of ice in source solutions: Primary and secondary nucleation. Ultrasonics, 2003, 41: 595-604

9 Swallowe G M, Field J E, Rees C S, et al. A photographic study of the effect of ultrasound on solidification. Acta Metall, 1989, 37(3): 961-967

10 Li J W, Momono T. Effect of ultrasonic output power on refining the crystal structures of ingots and its experimental simulation. J Mater Sci Technol, 2005, 21(1): 47-52

11 Campell J. Effect of vibration during solidification. Int Metall Rev, 1981, 26(2): 7-103

12 Ruan Y, Cao C D, Wei B B. Rapid growth of ternary eutectic under high undercooling conditions. Sci China Ser G-Phys Mech Astron, 2004, 47(6): 717-728

13 Baber B P, Putterman S J. Observation of synchronous picosecond sonoluminescence. Nature, 1991, 352: $318-320$

14 Hickling R. Transient high-pressure solidification associated with cavitation in water. Phys Rev Lett, 1994, 73: 2853-2855

15 Xie W J, Cao C D, Lü Y J, et al. Eutectic growth under acoustic levitation conditions. Phys Rev E, 2002, 66: 061601 